\title{
Correction to: Potentiometric Determination of Fluoride Concentration in Beers
}

\author{
Inmaculada Rodríguez ${ }^{1}$ - Juan Ramón Jaudenes ${ }^{1}$ - Arturo Hardisson ${ }^{2} \cdot$ Soraya Paz $^{1}$. \\ Carmen Rubio $^{1}$ - Angel José Gutiérrez ${ }^{1}$ (D) Antonio Burgos ${ }^{2} \cdot$ Consuelo Revert $^{3}$
}

Published online: 21 November 2017

(C) Springer Science+Business Media, LLC 2017, Corrected publication November/2017

Correction to: Biol Trace Elem Res (2017)

https://doi.org/10.1007/s12011-017-1029-8

The original version of the article unfortunately contained a mistake. The first author Inmaculada Rodríguez was omitted.

The authors regret the oversight.

The online version of the original article can be found https://doi.org/10. 1007/s12011-017-1029-8

$\triangle$ Angel José Gutiérrez

ajguti@ull.es

1 Área de Toxicología, Facultad de Ciencias de la Salud, Universidad de La Laguna, Campus de Ofra s/n, 38071 Santa Cruz de Tenerife, Spain

2 Área de Medicina Preventiva y Salud Pública, Facultad de Ciencias de la Salud, Universidad de la Laguna, Campus de Ofra s/n, 38071 Santa Cruz de Tenerife, Spain

3 Área de Fisioterapia, Facultad de Ciencias de la Salud, Universidad de La Laguna, Campus de Ofra s/n, 38071 Santa Cruz de Tenerife, Spain 